\title{
Incorrect administration method for booster dosage of rabies vaccination
}

Administração incorreta para vacinação anti rábica

Sir, the rabies vaccination is the best method for prevention of rabies, a deadly neurological infection. The problem of missed appointment of rabies vaccination is common and becomes an important concern in tropical neurology ${ }^{1}$. Here, the authors would like to share the experience on another important problem in clinical practice in maintenance of rabies vaccination schedule. The authors review the medical records of 250 booster dosages of rabies vaccination in our center in year 2013. Of overall 250 dosages, 230 were administered by intramuscular injection while the left 20 were administered by intradermal injection. However, when tracing back it can be seen that "incorrect administration method" can be seen in 11 cases (4.4\%). All detected cases were missed from intradermal administration to intramuscular administration. Since the administration of rabies vaccine can be by either intramuscular or intradermal injection, the practitioner, in charge nurse, can be easily miss administration and this can be problematic in prevention of disease. To provide knowledge to increase awareness of practitioner is required.

Beuy Joob ${ }^{1}$, Viroj Wiwanitkit ${ }^{2}$

\section{References}

1. Wiwanitkit V. Missed appointment in rabies vaccination schedule. Arq Neuropsiquiatr 2012;70:834. 